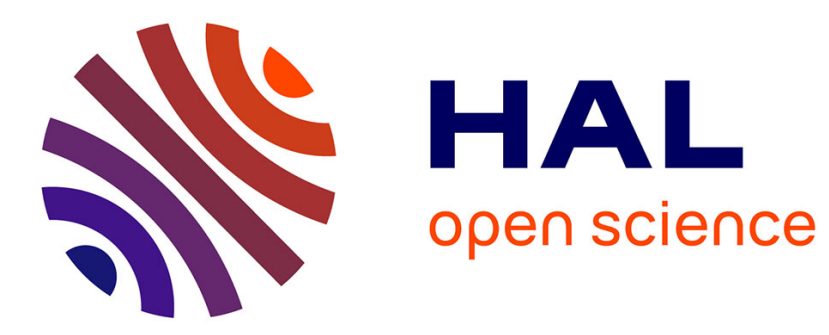

\title{
Marquage en fréquences d'un spectre hertzien
}

J. Legrand, B. Macke, J. Messelyn, R. Wertheimer

\section{To cite this version:}

J. Legrand, B. Macke, J. Messelyn, R. Wertheimer. Marquage en fréquences d'un spectre hertzien. Revue de Physique Appliquée, 1968, 3 (2), pp.199-202. 10.1051/rphysap:0196800302019900 . jpa00242845

\section{HAL Id: jpa-00242845 https://hal.science/jpa-00242845}

Submitted on 1 Jan 1968

HAL is a multi-disciplinary open access archive for the deposit and dissemination of scientific research documents, whether they are published or not. The documents may come from teaching and research institutions in France or abroad, or from public or private research centers.
L'archive ouverte pluridisciplinaire HAL, est destinée au dépôt et à la diffusion de documents scientifiques de niveau recherche, publiés ou non, émanant des établissements d'enseignement et de recherche français ou étrangers, des laboratoires publics ou privés. 


\title{
MARQUAGE EN FRÉQUENGES D'UN SPEGTRE HERTZIEN
}

\author{
Par J. LEGRAND, B. MACKE, J. MESSELYN et R. WERTHEIMER (1), \\ Laboratoire de Spectroscopie Hertzienne, Département de Physique, Bât. P $_{5}$, Faculté des Sciences de Lille, \\ Équipe de recherche associée au C.N.R.S.
}

(Reçu le 11 janvier 1968.)

\begin{abstract}
Résumé. - Pour étudier un spectre hertzien $S_{\mathrm{p}}(v)$ dépendant d'un paramètre $p$, il est commode de faire figurer sur un même enregistrement les courbes obtenues pour diverses valeurs de $p$. Le marquage en fréquence d'un tel réseau pose le problème de la surcharge des enregistrements; nous l'avons résolu en effectuant celui-ci par des courtes levées de plume de l'enregistreur. Avec notre dispositif, chaque courbe du réseau est affectée de vingt blancs définissant une échelle linéaire de fréquence $\nu$.

Abstract. - The study of a microwave spectrum $S_{\mathrm{p}}(\nu)$ which depends upon a parameter $p$ is greatly facilitated if we can record on a single chart the curves which correspond at different values of $p$. The fact that one must introduce frequency markers in a such set of curves raises the problem overloading the record charts; we can solve this problem with the conversion of frequency markers into "record-penrise-up" of short duration. With our experimental set-up, twenty white spaces appear on each curve of the record set which define a linear frequency scale for $v$.
\end{abstract}

Introduction. - Dans les expériences de double résonance en spectroscopie hertzienne [1], l'absorption observée $A\left(\nu, \nu^{\prime}\right)$ est fonction des fréquences de sonde $v$ et de pompe $\nu^{\prime}$. Pour étudier de façon détaillée une telle absorption, nous réalisons deux types d'expériences distincts en balayant respectivement les fréquences de pompe et de sonde pour diverses valeurs de l'autre fréquence jouant le rôle de paramètre. Dans les deux cas, par une méthode de transposition liée à l'utilisation de stabilisation de phase des sources hyperfréquences [2], le problème se ramène à la mesure d'une fréquence wobulée $F$ au voisinage de $10 \mathrm{MHz}$. Par exemple, si nous balayons $\nu^{\prime}$ fréquence de pompe, dans nos expériences celle-ci est liée à la fréquence $F$ par la relation :

$$
\nu^{\prime}=68430+54 F \quad(\mathrm{MHz}) .
$$

La wobulation de $F$ étant effectuée par diode varicap, sa linéarité est médiocre. Nous avons donc choisi une densité de marqueurs assez grande, soit vingt marqueurs sur l'intervalle de fréquence exploré. Par ailleurs, la durée d'enregistrement d'une courbe est au minimum de 50 s et un réseau comporte une moyenne de dix courbes. La fréquence $F$ étant délivrée par un oscillateur libre, il était impossible d'enregistrer

(1) Travail effectué sous contrat D.R.M.E. une échelle unique de marqueurs par réseau, car on craignait une dérive trop importante de la fréquence $F$ sur un intervalle de temps de $500 \mathrm{~s}$ au minimum. Pratiquement, il est apparu nécessaire de réaliser une mesure permanente de la fréquence $F$ et un marquage de chaque courbe au moment même de son enregistrement. Pour éviter la surcharge d'enregistrements parfois très denses, nous effectuons ce marquage en " modulation $Z$ ». Le passage d'un marqueur amène une levée électrique de la plume de l'enregistreur et se traduit par un blanc de la courbe enregistrée.

I. Principe. - La figure 1 donne le schéma synoptique du fréquencemètre. Le signal issu d'un pilote à quartz de fréquence $F_{\mathrm{Q}}$ est amplifié puis modulé en amplitude par un générateur de basse fréquence $2 n_{0}$. Le modulateur joue également le rôle d'écrêteur avec un angle d'ouverture de $60^{\circ}$ environ.

A la sortie de ce modulateur, on dispose ainsi d'un signal présentant des composantes spectrales de fréquence $F_{\mathbf{Q}}+k .2 n_{0}$ ( $k$ entier positif, nul ou négatif). Avec l'angle d'ouverture choisi, les cinquièmes bandes latérales $(k= \pm 5)$ ont une amplitude égale au moins au tiers de celle de la composante centrale $\left(F_{\mathrm{Q}}\right)$. Un mélangeur à diode délivre ensuite le battement entre ce spectre et la fréquence balayée $F$ à mesurer, chacun de ces signaux étant séparément 


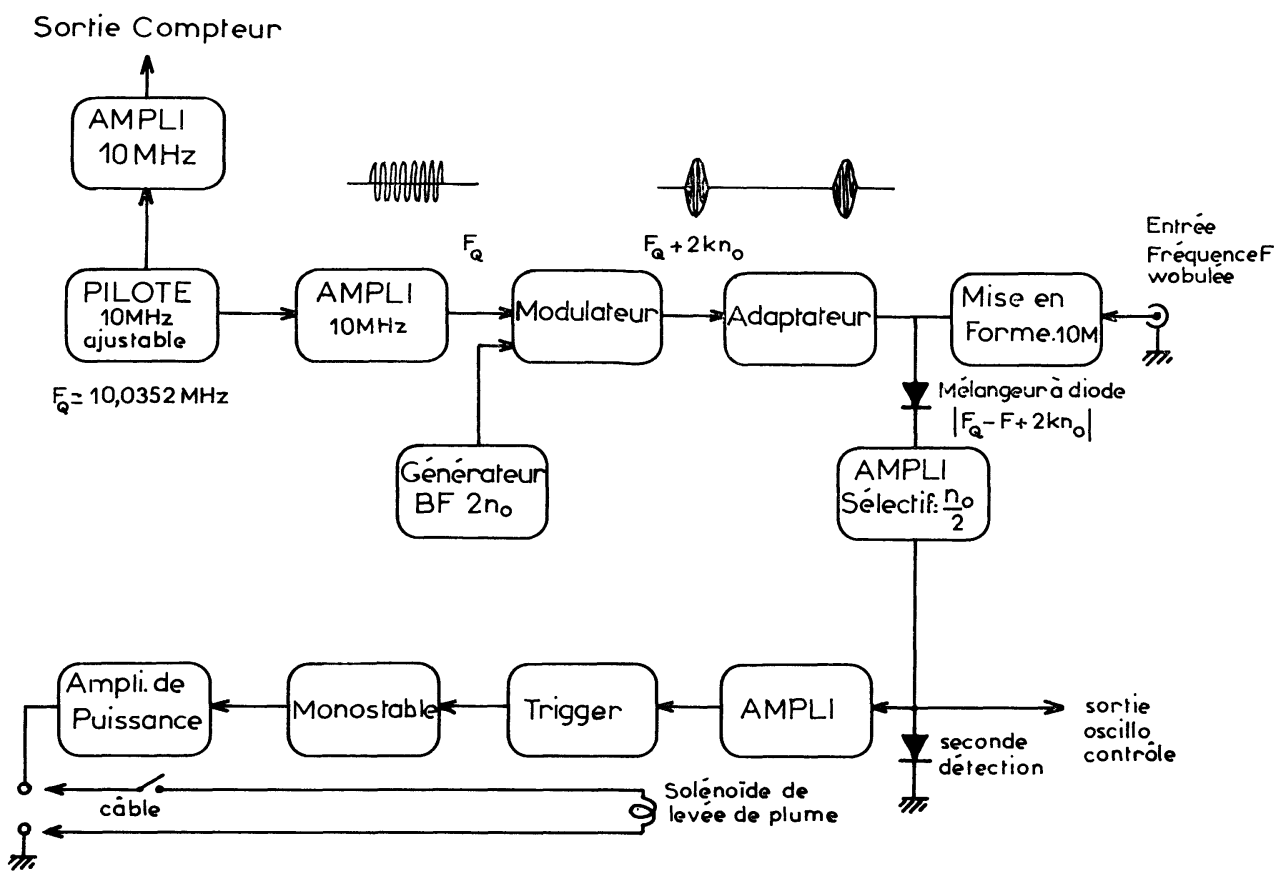

FIG. 1. - Schéma synoptique.

adapté aux caractéristiques du mélangeur. Ces mises en forme sont nécessaires pour éliminer les marqueurs parasites qui résulteraient de battements entre spectres harmoniques. Il faut également éviter que le mélangeur n'engendre les harmoniques du battement principal qui auraient le même effet. Comme dans un changeur de fréquence, les deux signaux doivent être d'amplitudes assez différentes. Nous avantageons dans un rapport dix environ la fréquence balayée. Moyennant ces précautions et un choix convenable de la constante de temps de détection, les seules fréquences disponibles à la sortie du mélangeur sont de la forme :

$$
\left|F-F_{Q}+2 k n_{0}\right| \text {. }
$$

Ce spectre est appliqué à un amplificateur sélectif accordé sur la fréquence $n_{0} / 2$ et suivi d'une deuxième détection. Ce "récepteur » délivre une impulsion chaque fois que la fréquence $F$ balayée passe sur l'une des valeurs :

$$
F=F_{\mathrm{Q}} \pm \frac{n_{0}}{2}+2 k n_{0} .
$$

On dispose ainsi d'une distribution linéaire de marqueurs distants de $n_{0}$. Ces marqueurs sont amplifiés et mis en forme par un " trigger de Schmitt» dont le seuil de déclenchement est tel qu'il ne transmet que les vingt marqueurs centraux (dix de part et d'autre de $F_{\mathrm{Q}}$ ). Ils sont alors appliqués à un monostable qui les transforme en impulsions rectangulaires de largeur réglable. Ceci permet d'ajuster la durée $T$ de levée à la vitesse de balayage; une levée trop courte n'est pas visible sur la courbe enregistrée et une levée trop longue introduit une incertitude. Le monostable est suivi d'un amplificateur de puissance qui fournit l'excitation convenable au solénoïde assurant la levée électrique de plume.

II. Réalisation pratique. - La fréquence choisie pour l'oscillateur à quartz est de 10,0352 MHz. Elle est ajustable autour de cette valeur. Un étage séparateur permet d'attaquer un compteur (Rochar A 1149) pour la mesure précise de la fréquence. On dispose de deux distributions de marqueurs, la distance entre marqueurs successifs étant de 3,7 ou $14,8 \mathrm{kHz}$. Un inverseur assure la commutation simultanée de la fréquence de modulation $(7,4$ ou $29,6 \mathrm{kHz})$ et de l'amplificateur sélectif $(1,85$ ou $7,4 \mathrm{kHz})$. Indiquons que le générateur basse fréquence de modulation et l'amplificateur sélectif sont de même conception; ils ne diffèrent que par le taux de réaction. De plus, le générateur $\mathrm{BF}$ comporte une régulation de niveau à thermistance qui maintient sa tension de sortie à $3,5 \mathrm{~V}$ crête à crête.

Cette condition de stabilité du niveau est indispensable à un fonctionnement correct du modulateur ( fig. 2). Celui-ci est constitué par un transistor dont la base est attaquée simultanément par les signaux HF et $\mathrm{BF}$. Le dosage relatif de ces signaux s'effectue en ajustant le niveau de la haute fréquence par le rhéostat de $1 \mathrm{k} \Omega$. Le réglage de l'angle d'ouverture est obtenu en ajustant la polarisation d'émetteur au moyen du rhéostat de $10 \mathrm{k} \Omega$. Le contacteur $\mathrm{C}$ permet de mettre le générateur BF hors service (cf. § III). Cette opération s'accompagne d'une modification convenable 


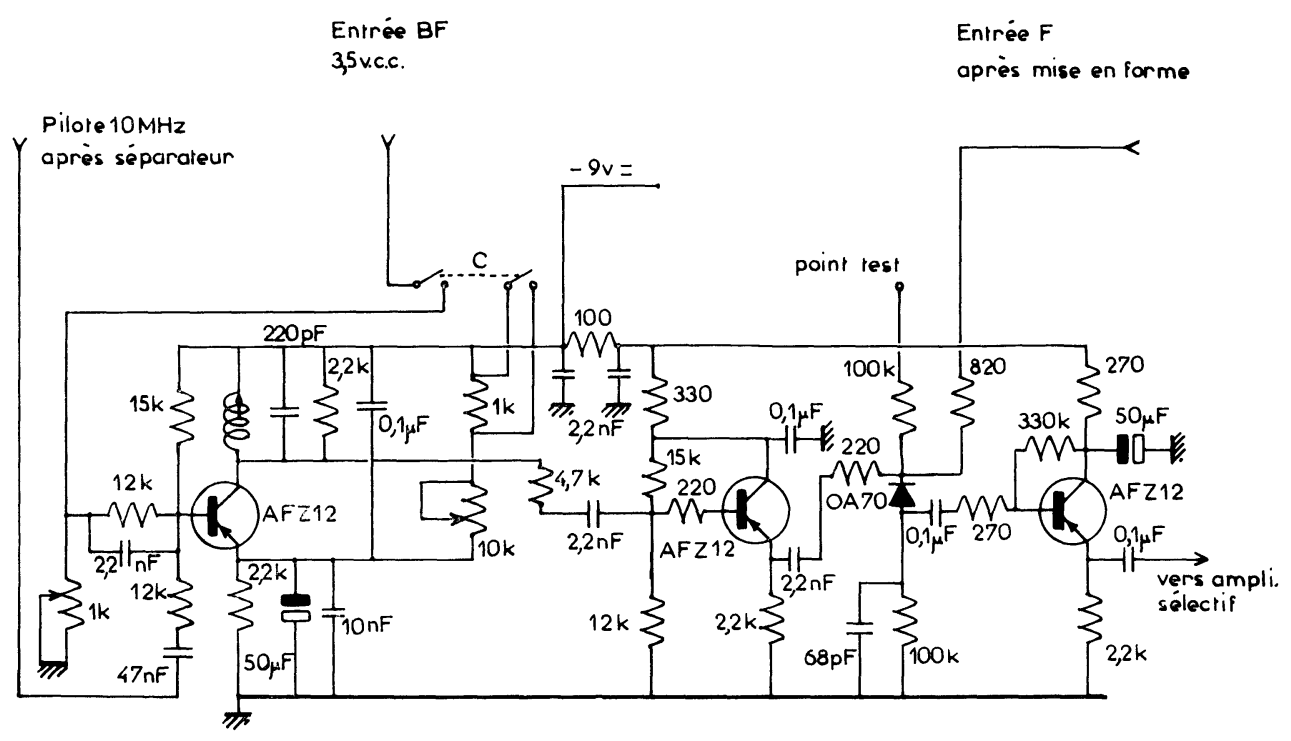

FIG. 2. - Modulateur et mélangeur.

de la polarisation d'émetteur qui ramène le niveau de la haute fréquence disponible sur le collecteur au niveau moyen des composantes obtenues lorsque le générateur BF est en service.

Le séparateur, le mélangeur et l'amplificateur sélectif ne présentent pas de particularités remarquables. L'amplificateur de remise en forme et le circuit monostable sont constitués par des modules commerciaux (Transco R.T.G. PS1 et OS1). La durée de levée de plume est choisie parmi les valeurs 0,1 , $0,2,0,3,0,5,0,7,1 \mathrm{~s}$. L'interrupteur électronique final est constitué par un transistor R.C.A. 2N 3055. Avec l'enregistreur graphique utilisé (Hewlett Packard $7035 \mathrm{AM}$ ), les conditions d'excitation du solénoïde de levée de plume sont les suivantes :

Plume levée : courant : $125 \mathrm{~mA}$; tension inférieure à $5 \mathrm{~V}$;

Plume baissée : courant inférieur à $10 \mathrm{~mA}$; tension $47 \mathrm{~V}$.

III. Utilisation et performances. - Il est indispensable dans l'utilisation de ce matériel de pouvoir définir la fréquence centrale de l'enregistrement correspondant à $F$ égale à $F_{\mathrm{Q}}$. A cet effet, on met hors circuit le générateur BF. On n'observe alors que deux marqueurs correspondant aux fréquences $F=F_{\mathrm{Q}} \pm \frac{n_{0}}{2}$.

Pour vérifier que la distribution de marqueurs est correcte, on dispose d'une sortie de contrôle à la sortie de l'amplificateur sélectif. En appliquant une fréquence fixe réglable à l'entrée $F$, on compte trois valeurs successives de cette fréquence, $F_{1}, F_{2}$ et $F_{3}$, pour lesquelles on observe un accord. $F_{3}-F_{1}$ fournit la fréquence du générateur $\mathrm{BF}$ que l'on ajuste à la valeur exacte. On accorde ensuite l'amplificateur sélectif pour amener $F_{2}$ à la moyenne arithmétique de $F_{1}$ et $F_{3}$.

Les performances de l'appareil sont liées à la stabilité des fréquences des oscillateurs $\mathrm{HF}$ et $\mathrm{BF}$ et à la précision du déclenchement de la levée de plume. La dérive de l'oscillateur à quartz est inférieure à $100 \mathrm{~Hz}$. Elle introduit une translation d'ensemble des marqueurs qui, dans le cas le plus défavorable, n'est que d'un tiers de millimètre (enregistrement de format utile $10 \times 25 \mathrm{~cm}$ ). La stabilité de l'oscillateur et de l'amplificateur sélectif basse fréquence peut être estimée à $0,2 \%$. Ceci correspond, pour les marqueurs marginaux, à un déplacement de l'ordre de $0,2 \mathrm{~mm}$. Les dérives de fréquences n'introduisent donc pas d'erreur appréciable.

La précision du déclenchement de la levée de plume est fonction de la vitesse de balayage et de la distribution d'amplitude des marqueurs. On est limité en vitesse de balayage par les temps de réponse des amplificateurs sélectifs (inversement proportionnels à leur bande passante) et du dispositif électromécanique de levée de plume (de l'ordre de $20 \mathrm{~ms}$ ). Le seuil de déclenchement de l'électronique de commutation étant fixe, l'inégalité d'amplitude des différents marqueurs se traduit par un léger retard des levées de plume associées aux marqueurs les plus faibles.

Pour vérifier que ces défauts n'avaient pas un caractère rédhibitoire, nous avons effectué un enregistrement test ( fig. 3). En voie $\mathrm{X}$ est appliqué un signal de balayage synchrone du signal de wobulation de la fréquence $F$; en voie $\mathrm{Y}$, nous appliquons la tension obtenue après la seconde détection (sortie oscillo, contrôle de la figure 1). Les enregistrements (1) et (2) 


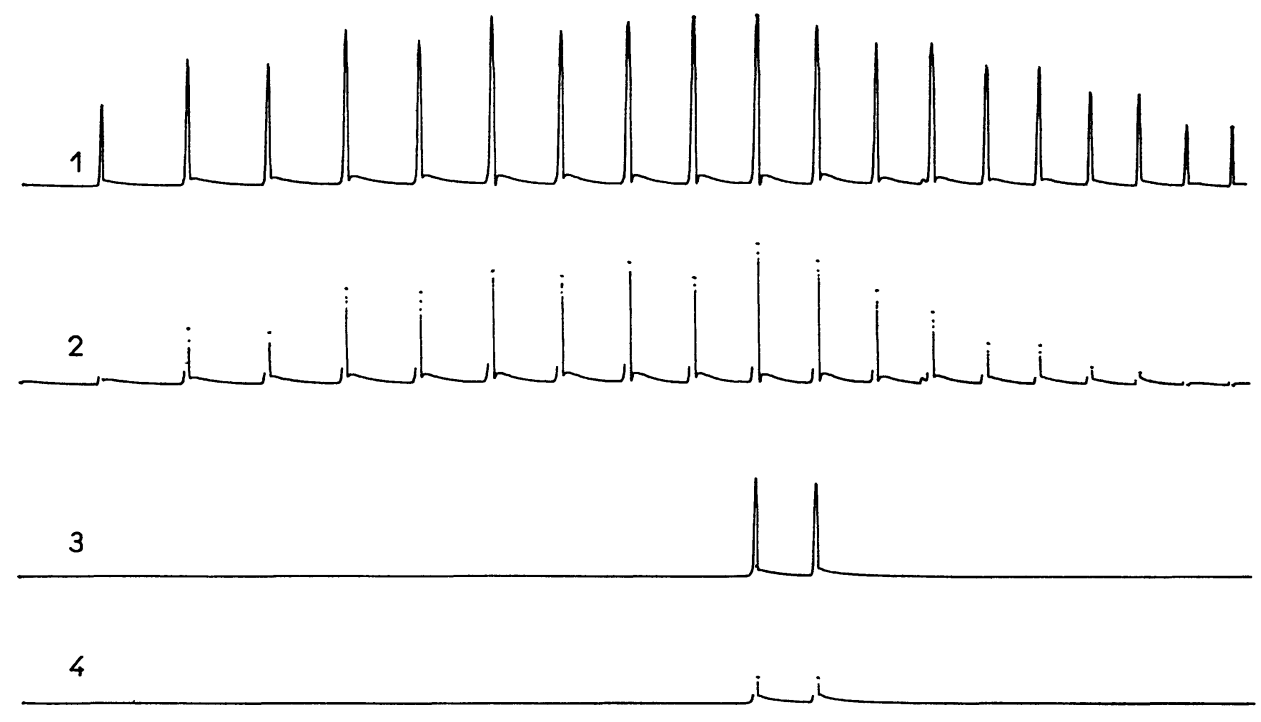

FIG. 3. - Enregistrement test.

sont obtenus respectivement sans et avec levée de plume; les enregistrements (3) et (4) leur correspondent lorsque l'oscillateur BF est mis hors service (marqueurs centraux). L'écart maximum entre la pointe du marqueur et le centre de la levée de plume est de $0,5 \mathrm{~mm}$, c'est-à-dire parfaitement acceptable.

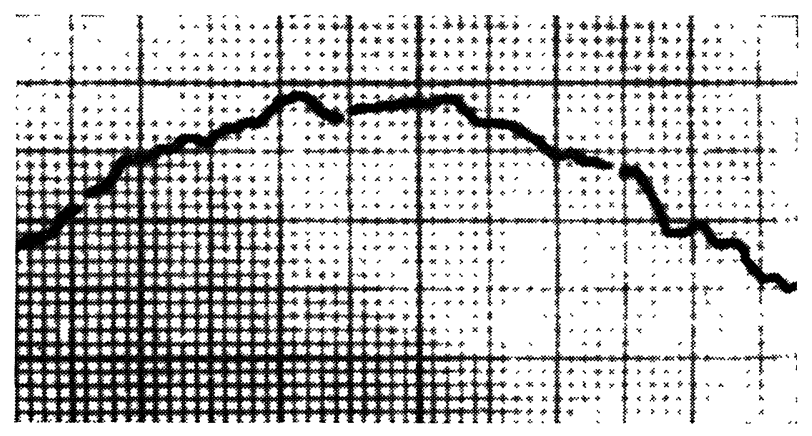

FIG. 4. - Détail d'enregistrement.
La figure 4 donne un détail d'enregistrement marqué en fréquence à l'aide de notre appareillage et montre sa souplesse d'emploi.

Conclusion. - Pour un usage particulier, nous avons réalisé un fréquencemètre d'utilisation assez générale. En l'adaptant à chaque problème particulier, il permet un marquage commode en fréquence de tout spectre obtenu en fréquence wobulée. On pourrait améliorer éventuellement la précision du déclenchement en réalisant une distribution de marqueurs à amplitude constante. Ceci peut être obtenu en utilisant des circuits surcouplés ou à accords décalés qui avantageraient les marqueurs marginaux. Le fonctionnement particulier du modulateur ne permet pas d'obtenir ce résultat en agissant sur le spectre basse fréquence.

Remerciements. - Les auteurs remercient MM. Boudy et Rosseels pour leur collaboration technique.

\section{BIBLIOGRAPHIE}

[1] Legrand (J.), Macke (B.) et Messelyn (J.), $C . R$. Acad. Sc., 1967, 264, 60.

[2] Macke (B.), Messeiyn (J). et Wertheimer (R.), Onde électrique, 1966, 46, 123. 